\title{
Nanocrystalline glass-coated FeNiMoB microwires
}

\author{
E. Komova, ${ }^{1}$ M. Varga,${ }^{2}$ R. Varga, ${ }^{2, a)}$ P. Vojtanik, ${ }^{2}$ J. Bednarcik, ${ }^{3}$ J. Kovac, ${ }^{4}$ M. Provencio, ${ }^{5}$ \\ and M. Vazquez ${ }^{5}$ \\ ${ }^{1}$ Faculty of Aeronautics, TU Kosice, Rampova 7, 04121 Kosice, Slovakia \\ ${ }^{2}$ Faculty of Science, Institute of Physics, Park Angelinum 9, 04154 Kosice, Slovakia \\ ${ }^{3}$ HASYLAB am DESY, Notkestrasse 85, D-22603 Hamburg, Germany \\ ${ }^{4}$ Institute of Experimental Physics, SAS, Watsonova 47, 04353 Kosice, Slovakia \\ ${ }^{5}$ Institute of Materials Science, CSIC, 28049 Madrid, Spain
}

(Received 30 May 2008; accepted 16 July 2008; published online 12 August 2008)

\begin{abstract}
The evolution of the structure of glass-coated $\mathrm{Fe}_{40} \mathrm{Ni}_{38} \mathrm{Mo}_{4} \mathrm{~B}_{16}$ amorphous microwire with thermal treatments and its interplay with magnetism has been studied. As shown by $\mathrm{x}$-ray diffraction, a primary crystallization process resulted into formation of $\gamma-(\mathrm{Fe}, \mathrm{Ni})$ nanocrystallites embedded in a residual amorphous matrix. The evolution of the saturation magnetization and the switching field after different thermal treatment was studied. Amorphous glass-coated microwires based on FeNi exhibit magnetic bistability even in the nanocrystalline state. This is explained by the high magnetoelastic anisotropy, which is also responsible for magnetic hardening after annealing at the temperatures above 670 K. (C) 2008 American Institute of Physics. [DOI: 10.1063/1.2969057]
\end{abstract}

Amorphous and nanocrystalline ferromagnetic microwires covered by a glass coating are very attractive material for applications, mainly as sensing elements in various sensors (i.e., magnetic field, coding, etc.), due to their outstanding magnetic characteristics, small dimensions and reliability on electrical, mechanical, and corrosive external effects (as a result of the Pyrex coating). ${ }^{1}$ These microwires, prepared by quenching and drawing technique, consist of a magnetic nucleus (with diameter from 1-30 $\mu \mathrm{m}$ ) coated by a Pyrexlike glass (thickness of $2-20 \mu \mathrm{m}$ ). Due to their amorphous nature, magnetoelastic and shape anisotropy contributions govern their magnetic properties. As a result of abovementioned anisotropies, the domain structure of microwires with positive magnetostriction consists of single axial domain and their magnetization process is characteristic of the bistable behavior. ${ }^{1,2}$

The nanocrystalline microwires have been subject of different studies starting from Finemet based microwires, ${ }^{3-7}$ as well as the Nanoperm based ones. ${ }^{8}$ Such nanocrystalline microwires are usually prepared by heat treatment. However, the microwires undergo strong stresses during the annealing due to the different thermal expansion coefficients of the metallic nucleus and the glass coating. As a result, a $\gamma$-Fe phase appears in annealed glass-coated Finemet microwires instead of desired $\alpha-(\mathrm{Fe}, \mathrm{Si})$ phase. ${ }^{6,7}$ When the annealing is performed under high pressure, the formation of $\gamma$-Fe phase is energetically more favorable due to its higher pack density, However, the $\gamma$-Fe phase is nonmagnetic and deteriorates the good soft magnetic properties of nanocrystalline microwires. ${ }^{6,7}$

It has been recently shown that partial crystallization of $\mathrm{Fe}_{40} \mathrm{Ni}_{38} \mathrm{Mo}_{4} \mathrm{~B}_{16}$ ribbon leads to the formation of nanocrystalline materials with $\gamma-(\mathrm{Fe}, \mathrm{Ni})$ nanocrystals having diameter of $10 \mathrm{~nm} .{ }^{9}$ Annealing of such samples at $T_{a}=700 \mathrm{~K}$ has been shown to be the optimum annealing temperature to obtain nanocrystalline $\mathrm{Fe}_{40} \mathrm{Ni}_{38} \mathrm{Mo}_{4} \mathrm{~B}_{16}$ alloy with optimized soft magnetic properties (lowest magnetostriction, lowest coercive field, and highest initial susceptibility). ${ }^{10,11}$

\footnotetext{
a) Author to whom correspondence should be addressed. Electronic mail: rvarga@upjs.sk.
}

Since $\gamma-(\mathrm{Fe}, \mathrm{Ni})$ phase is ferromagnetic contrary to the paramagnetic $\gamma$-Fe phase, it is a promising candidate for preparation of soft magnetic nanocrystalline microwires. We demonstrate that $\mathrm{Fe}_{40} \mathrm{Ni}_{38} \mathrm{Mo}_{4} \mathrm{~B}_{16}$ alloy composition can be considered to prepare soft magnetic nanocrystalline microwires with positive magnetostriction. Such microwires exhibit bistability even in the nanocrystalline state.

The study has been performed on glass-coated amorphous microwires with nominal composition of $\mathrm{Fe}_{40} \mathrm{Ni}_{38} \mathrm{Mo}_{4} \mathrm{~B}_{18}$ prepared by the Taylor-Ulitovsky method. ${ }^{12}$ The diameter of the metal core was $8 \mu \mathrm{m}$ and the total diameter $12 \mu \mathrm{m}$. In order to obtain nanocrystalline specimens, the amorphous microwires were annealed for $1 \mathrm{~h}$ at various temperatures ranging from 570 to $820 \mathrm{~K}$ in helium atmosphere. Microstructure of the annealed material was analyzed by $\mathrm{x}$-ray diffraction (XRD). High-energy XRD measurements were performed at HASYLAB at DESY (Hamburg, Germany) on the experimental station BW5 at DORIS storage ring using monochromatic synchrotron radiation of 100 $\operatorname{keV}(\lambda=0.12398 \AA)$. The samples measured at room temperature in the transmission mode were illuminated for $30 \mathrm{~s}$ by well collimated incident beam having a cross section of $1 \times 1 \mathrm{~mm}^{2}$. XRD patterns were recorded using a twodimensional (2D) detector (mar345 Image plate) in symmetric mode. The background intensity was subtracted directly from 2D XRD pattern, and the result was integrated to the $Q$ space $[Q=4 \pi \sin (\theta) / \lambda]$ using the software package FIT2D. ${ }^{13}$

The saturation magnetization was measured by vibrating-sample magnetometer at maximum applied field of $1 T$ in the temperature range of $4-800 \mathrm{~K}$. The heating and cooling rate was $10 \mathrm{~K} / \mathrm{min}$. The same sample was subsequently heated up to the given annealing temperature (570, $620,670,720$, and $770 \mathrm{~K}$, respectively). Then, the sample was annealed at this temperature for $1 \mathrm{~h}$ and finally it was cooled down.

The switching field has been measured at room temperature by the induction method, ${ }^{14}$ using triangular waveform at the frequency $100 \mathrm{~Hz}$.

As can be seen in Fig. 1, $\mathrm{Fe}_{40} \mathrm{Ni}_{38} \mathrm{Mo}_{4} \mathrm{~B}_{16}$ samples annealed for $1 \mathrm{~h}$ at temperatures below $620 \mathrm{~K}$ exhibit a diffuse scattering patterns typical for metallic glasses with a maxi- 


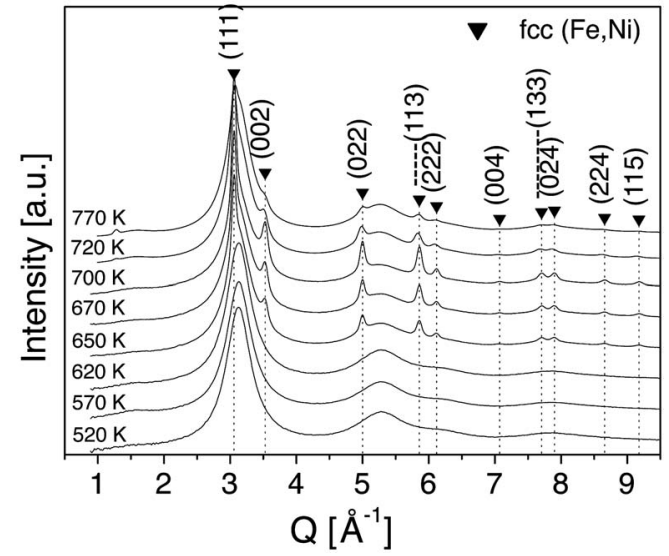

FIG. 1. XRD patterns corresponding to FeNiMoB microwires annealed for $1 \mathrm{~h}$ at given temperatures.

mum at $Q=3.13 \AA^{-1}$ and pronounced oscillations visible up to $Q=10 \AA^{-1}$. The second broad peak at $Q=5.28 \AA^{-1}$ has a shoulder at high- $Q$ side which is again a common feature of conventional metallic glasses. XRD patterns of samples heat treated above $620 \mathrm{~K}$ show already small and rather broad peaks of the $\gamma-(\mathrm{Fe}, \mathrm{Ni})$ phase in addition to the diffuse amorphous contribution. Refined cell parameter of $\gamma$ - $(\mathrm{Fe}, \mathrm{Ni})$ solid solution [space-group $F m-3 m(225)]$ is $a=3.556 \AA$. The intensity of observed peaks increases with the annealing temperature up to $700 \mathrm{~K}$. This indicates that initially amorphous $\mathrm{Fe}_{40} \mathrm{Ni}_{38} \mathrm{Mo}_{4} \mathrm{~B}_{16}$ alloy partially crystallize upon annealing. Rather broad peaks indicate the presence of nanocrystalline $\gamma-(\mathrm{Fe}, \mathrm{Ni})$ grains embedded in an amorphous matrix. It should be noted here that quantitative analysis of crystal size from line broadening failed due to fact that reflections belonging to fcc- $(\mathrm{Fe}, \mathrm{Ni})$ are rather broad and still overlapped by amorphous component making correct determination of linewidths difficult. On the other hand, taking into account limitations of XRD, we can conclude that nanocrystals have size certainly below $10 \mathrm{~nm}$. Another interesting feature of XRD patterns shown in Fig. 1 is the fact that further increase of annealing temperature above $700 \mathrm{~K}$ leads to vanishing of Bragg peaks. It probably results from an interplay between effect of temperature and increased pressure due to different thermal expansion coefficients of inner metallic and surrounding glass part, respectively. It has been shown $^{14}$ that the stresses $\sigma_{a}(T)$ arising from the different thermal expansion coefficient of metallic nucleus $\alpha_{m}$ and glass coating $\alpha_{g}$ are proportional to the temperature $T$,

$$
\sigma_{a}(T) \approx E\left(\alpha_{g}-\alpha_{m}\right) T
$$

where $E$ is Young modulus. As a result, very high stresses are induced on the metallic nucleus. Such stresses could reach up to $1 \mathrm{GPa}^{6}{ }^{6}$ which could result in the increase of the crystallization temperature as a result of the suppression of atomic mobility. ${ }^{15-17}$

Since partial crystallization of initially amorphous microwire results into formation of ferromagnetic fcc- $(\mathrm{Fe}, \mathrm{Ni})$ phase, its presence and temperature behavior can be also studied by measuring the temperature dependence of magnetization $M_{S}(T)$ (Fig. 2). The temperature dependence of magnetization for two-component systems is given as ${ }^{18}$

$$
M_{S}(T)=\nu_{\mathrm{am}} M_{\mathrm{am}}(T)+\nu_{\mathrm{cr}} M_{\mathrm{cr}}(T),
$$

where $\nu_{i}$ is the volume fractions and $M_{i}(T)$ is saturation mag-

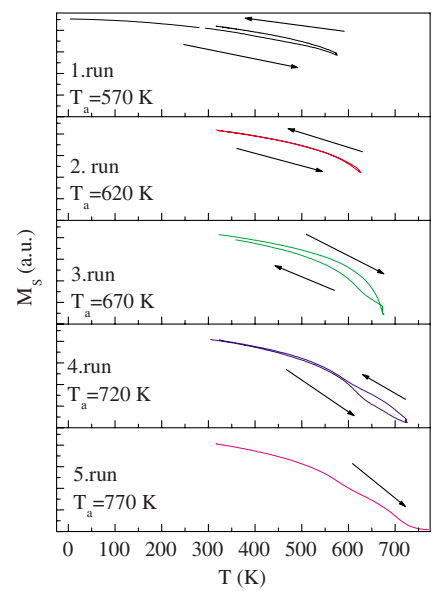

FIG. 2. (Color online) Thermomagnetic analysis of the annealed $\mathrm{Fe}_{40} \mathrm{Ni}_{38} \mathrm{Mo}_{4} \mathrm{~B}_{16}$ microwire.

netizations of the two magnetic phases $[i=$ amorphous (crystalline)].

Thermomagnetic analysis of the devitrification process is shown in Fig. 2. The Curie temperatures of the amorphous $T_{C}(\mathrm{am})$ and crystalline $T_{C}(\mathrm{cr})$ phases were obtained by the analysis of the thermomagnetic curves using Eq. (2). The results of quantitative analysis are collected in Table I.

Annealing at $670 \mathrm{~K}$ leads to the crystallization of $\gamma$ - $(\mathrm{Fe}, \mathrm{Ni})$ phase, which is accompanied with a decrease of the Curie temperature of the amorphous phase, since the concentration of $\mathrm{Fe}$ and $\mathrm{Ni}$ in the amorphous phase decreases. The Curie temperature of the crystalline phase was found to be $739 \mathrm{~K}$. Contrary to the x-ray measurements, where annealing above $700 \mathrm{~K}$ leads to the disappearance of the crystalline phase, the thermomagnetic analysis shows further increase of the crystalline volume fraction that is accompanied by the increase of the magnetization above the Curie temperature of the amorphous phase. The Curie temperature of the amorphous phase is not affected by the further annealing; however, the Curie temperature of the crystalline grains decreases slightly. The difference between x-ray and thermomagnetic measurements could be explained by the fact that in the case of magnetization measurements, the same sample is used. Hence, the sample is already nanocrystalline when it is submitted to the annealing process at the temperature above $700 \mathrm{~K}$.

Switching field in bistable microwires is mainly governed by the magnetoelastic interaction of the magnetic moments and the stresses introduced during the microwire production. ${ }^{14}$ Annealing at low temperatures causes partial relaxation of the internal stresses introduced during microwires production. This results in the decrease of the switching field (Fig. 3).

Moreover, the magnetostriction of amorphous $\mathrm{Fe}_{40} \mathrm{Ni}_{38} \mathrm{Mo}_{4} \mathrm{~B}_{16}$ alloys decreases by annealing too. ${ }^{10}$ One can see that the values of minimum switching field $H_{\text {sw }}$ was

TABLE I. The Curie temperatures of amorphous $T_{C}(\mathrm{am})$ and crystalline $T_{C}$ (cr) phase of the $\mathrm{Fe}_{40} \mathrm{Ni}_{38} \mathrm{Mo}_{4} \mathrm{~B}_{16}$ microwire after different thermal treatments.

\begin{tabular}{cccccc}
\hline \hline$T_{a}(K)$ & 570 & 620 & 670 & 720 & 770 \\
\hline$T_{C(\mathrm{am})}(K)$ & 696 & 672 & 621 & 623 & 622 \\
$T_{C(\mathrm{crr})}(K)$ & & & 739 & 735 & 724 \\
\hline \hline
\end{tabular}




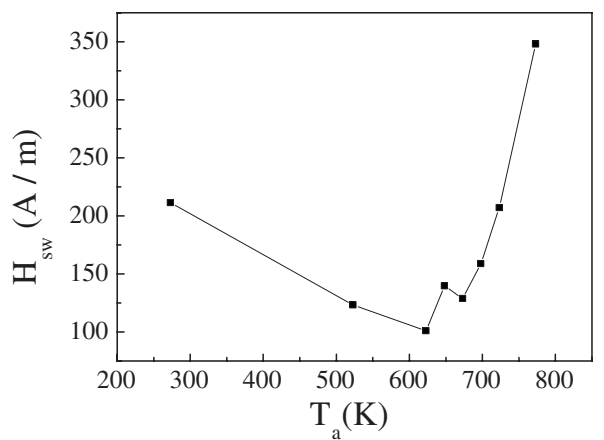

FIG. 3. The dependence of the switching field $H_{\mathrm{sw}}$ on the annealing temperature $T_{a}$.

reached at $T_{a}=620 \mathrm{~K} / 1 \mathrm{~h}$. Annealing above $620 \mathrm{~K}$, the crystalline $\gamma-(\mathrm{Fe}, \mathrm{Ni})$ phase appears. In the case of nanocrystalline materials, the coercivity depends on the exchange interaction of the crystalline grains. ${ }^{18}$ In the early stage of crystallization, when the diameter of the grains is too small and the distance between them is longer then the exchange length, the interaction is smooth. The precipitates plays the role of pinning centers for the domain walls and the switching field slightly increases. This happens after annealing at $650 \mathrm{~K}$. Further annealing leads to the increase of the crystalline $\gamma-(\mathrm{Fe}, \mathrm{Ni})$ grains diameter and the distance between them decreases. Because of the random crystallographic orientation of the grains, the overall magnetocrystalline anisotropy of the grains is averaged out. The decrease of anisotropy results in the decrease of the switching field after annealing at temperature $670 \mathrm{~K}$.

After treating at the temperatures above $T_{a}$ $=670 \mathrm{~K} / 1 \mathrm{~h}$, a steep increase of the switching field was detected, which can be interpreted as the magnetic hardening of $\mathrm{Fe}_{40} \mathrm{Ni}_{38} \mathrm{Mo}_{4} \mathrm{~B}_{16}$ alloy. In a case of nanocrystalline materials, it is a typically a result of the crystalline grains diameter increase. According to Herzer's theoretical model, ${ }^{19}$ an exchange coupling between such nanocrystalline grains can be effective in suppressing magnetocrystalline anisotropy $K_{1}$ of the individual grains. Hence the coercivity $H_{c}$ fulfill the sixth power law dependence on the crystalline grains diameter $D\left(H_{c} \sim D^{6}\right)$.

However, the crystallization is suppressed during the annealing above $670 \mathrm{~K}$ in FeNiMoB glass-coated microwire (see $\mathrm{x}$-ray results). Therefore, another mechanism must be responsible for the increase of the coercivity. It was shown by Suzuki et al. $^{20}$ that the effective anisotropy $\langle K\rangle$ in some nanocrystalline materials is given not only by the random local magnetocrystalline anisotropy $K_{1}$ [as given by Eq. (3)], but induced anisotropy $K_{u}$ should also be taken into account:

$$
\langle K\rangle=\left(\left\langle K_{1}\right\rangle^{2}+K_{u}^{2}\right)^{1 / 2} .
$$

The induced anisotropy $K_{u}$ is determined by the magnetoelastic anisotropy and is much stronger than $\left\langle K_{1}\right\rangle$. In the case of glass-coated microwires, strong stresses are applied on metallic nucleus during annealing at high temperature by the glass coating. Moreover, the saturation magnetostriction of FeNiMoB alloys increases by annealing at higher than optimal temperature. ${ }^{10,11}$ Hence, the magnetoelastic anisotropy increases and the switching field does the same too.

To conclude, we have studied the nanocrystalline glasscoated microwires based on $\mathrm{Fe}_{40} \mathrm{Ni}_{38} \mathrm{Mo}_{4} \mathrm{~B}_{16}$ composition. Its nanocrystalline structure consists of crystalline $\gamma-(\mathrm{Fe}, \mathrm{Ni})$ grains embedded in the amorphous residual structure. The formation of the $\gamma-(\mathrm{Fe}, \mathrm{Ni})$ ferromagnetic phase is more favorable than for other alloy compositions in the case of glass-coated microwire due to its higher packing density. We show that annealing at optimum temperature $(670 \mathrm{~K})$ leads to the magnetic softening. The annealing at temperatures above $670 \mathrm{~K}$ leads to a final magnetic hardening typical of fully crystallized samples. However, this is described in terms of the increase of the magnetoelastic anisotropy in contrary to the Finemet based alloys, where increase of the grains diameter is responsible. Moreover, it should be emphasized that glass-coated FeNiMoB microwires shows bistability in amorphous as well as nanocrystalline state. This feature, together with high structural and magnetic stability of nanocrystalline microwires makes them very promising materials for potential application in sensors.

This work was supported by Slovak Ministry of Education under APVT Grant Nos. APVT-20-007804, VEGA-1/ 3035/06, and MVTS 6RP/Manunet/UPJS/08.

${ }^{1}$ M. Vazquez, in Handbook of Magnetism and Advanced Magnetic Materials, edited by H. Kronmüller and S. Parkin (Wiley, Chichester, 2007), p. 221.

${ }^{2}$ H. Chiriac and T. A. Ovari, Prog. Mater. Sci. 40, 333 (1996).

${ }^{3}$ J. Arcas, C. Gómez-Polo, A. Zhukov, and M. Vázquez, Nanostruct. Mater. 7, 823 (1996).

${ }^{4}$ H. Chiriac, T. A. Ovari, G. Pop, and F. Barariu, J. Appl. Phys. 81, 5817 (1997).

${ }^{5}$ C. Dudek, A. L. Adenot-Engelvin, F. Bertin, and O. Acher, J. Non-Cryst. Solids 353, 925 (2007)

${ }_{6}^{6}$ J. Gonzalez, A. Zhukov, V. Zhukova, A. F. Cobeno, J. M. Blanco, A. R. de Arellano-Lopez, S. Lopez-Pombero, J. Martinez-Fernandez, V. Larin, and A. Torcunov, IEEE Trans. Magn. 36, 3015 (2000).

${ }^{7}$ R. Varga, C. Luna, A. Zhukov, M. Vazquez, and P. Vojtanik, Czech. J. Phys. 54, 177 (2004).

${ }^{8}$ C. Garcia, A. Zhukov, J. Gonzalez, V. Zhukova, R. Varga, J. J. del Val, V. Larin, A. Chizhik, and J. M. Blanco, J. Appl. Phys. 99, 08F116 (2006).

${ }^{9}$ R. V. Ramanujan and S. W. Du, J. Alloys Compd. 425, 251 (2006).

${ }^{10}$ R. Andrejco, R. Varga, P. Marko, and P. Vojtanik, Czech. J. Phys. 52, A113 (2002).

${ }^{11}$ J. Li, Z. Su, F. Wei, Z. Yang, H. Hahn, T. Wang, and S. Ge, Chin. Phys. Lett. 16, 211 (1999).

${ }^{12}$ G. F. Taylor, Phys. Rev. 23, 655 (1924).

${ }^{13}$ A. P. Hammersley, S. O. Svensson, M. Hanfland, A. N. Fitch, and D. Häusetmann, High Press. Res. 14, 235 (1996).

${ }^{14}$ R. Varga, K. L. Garcia, A. Zhukov, M. Vazquez, and P. Vojtanik, Appl. Phys. Lett. 83, 2620 (2003).

${ }^{15}$ J. Z. Jiang, T. J. Zhou, H. Rasmussen, U. Kuhn, J. Eckert, and C. Lathe, Appl. Phys. Lett. 77, 3553 (2000).

${ }^{16}$ J. Bednarcik, R. Nicula, M. Stir, C. Lathe, and E. Burkel, J. Non-Cryst. Solids 353, 862 (2007)

${ }^{17}$ W. H. Wang, P. Wen, D. Q. Zhao, M. X. Pan, T. Okada, and W. Utsumi, Appl. Phys. Lett. 83, 5202 (2003).

${ }^{18}$ G. Herzer, IEEE Trans. Magn. 25, 3327 (1989).

${ }^{19}$ G. Herzer, IEEE Trans. Magn. 26, 1397 (1990).

${ }^{20}$ K. Suzuki, N. Ito, S. Saranu, U. Herr, A. Michels, and J. S. Garitaonandia, J. Appl. Phys. 103, 07E730 (2008) 